\title{
MitoWave: Spatio-temporal analysis of mitochondrial membrane potential fluctuations during ischemia- reperfusion
}

D. Ashok, M.S., and B. O'Rourke, Ph.D.

*To whom correspondence should be addressed

Short title: Analysis of Mitochondrial Oscillation

Keywords: oscillation, oxidative phosphorylation, ischemia, reperfusion, wavelet, image processing, time-series analysis, mitochondrial membrane potential

Conflict of interest: The authors have no conflicts to disclose 


\begin{abstract}
:
Mitochondria exhibit non-stationary unstable membrane potential $\left(\Delta \Psi_{\mathrm{m}}\right)$ when subjected to stress, such as during Ischemia/Reperfusion (I/R). Understanding the mechanism of $\Delta \Psi_{m}$ instability involves characterizing and quantifying this phenomenon in response to $\mathrm{I} / \mathrm{R}$ stress in an unbiased and reproducible manner. We designed a simple ImageJ-MATLAB-based workflow called 'MitoWave' to unravel dynamic mitochondrial $\Delta \Psi_{\mathrm{m}}$ changes that occur during ischemia and reperfusion. MitoWave employs MATLAB's wavelet transform toolbox. In-vitro Ischemia was effected by placing a glass coverslip for 60 minutes on a monolayer of neonatal mouse ventricular myocytes (NMVMs). Removal of the coverslip allowed for reperfusion. $\Delta \Psi_{\mathrm{m}}$ response to I/R was recorded on a confocal microscope using TMRM as the indicator. As proof-of-principle, we used MitoWave analysis on ten invitro I/R experiments. Visual observations corroborated quantitative MitoWave analysis results in classifying the ten $I / R$ experiments into five outcomes that were observed based on the oscillatory state of $\Delta \Psi_{m}$ throughout the reperfusion time period. Statistical analysis of the distribution of oscillating mitochondrial clusters during reperfusion shows significant differences between five different outcomes $(p<0.001)$. Features such as time-points of $\Delta \Psi_{m}$ depolarization during $\mathrm{I} / \mathrm{R}$, area of mitochondrial clusters and time-resolved frequency components during reperfusion were determined per cell and per mitochondrial cluster. We found that mitochondria from NMVMs subjected to I/R oscillate in the frequency range of $8.6-45 \mathrm{mHz}$, with a mean of $8.73 \pm 4.35 \mathrm{mHz}$. Oscillating clusters had smaller areas ranging from $49.78 \pm 40.64 \mu \mathrm{m}^{2}$ while non-oscillating clusters had larger areas $65.97 \pm 42.07 \mu \mathrm{m}^{2}$. A negative correlation between frequency and mitochondrial cluster area was seen. We also observed that late $\Delta \Psi_{\mathrm{m}}$ loss during ischemia correlated with early $\Delta \Psi_{m}$ stabilization after oscillation on reperfusion. Thus, MitoWave analysis provides a way to quantify complex time-resolved mitochondrial behavior. It provides an easy to follow workflow to automate microscopy analysis and allows for unbiased, reproducible quantitation of complex nonstationary cellular phenomena.
\end{abstract}

\title{
Statement of Significance:
}

Understanding mitochondrial instability in Ischemia Reperfusion injury is key to determining efficacy of interventions. The MitoWave analysis is a powerful yet simple tool that enables even beginner MATALAB-Image $J$ users to automate analysis of time-series from microscopy data. While we used it to detect $\Delta \Psi_{m}$ changes during $\mathrm{I} / \mathrm{R}$, it can be adapted to detect any such spatio-temporal changes. It standardizes the quantitative analysis of complex biological signals, opens the door to in-depth screening of the genes, proteins and mechanisms underlying metabolic recovery after ischemia-reperfusion. 


\section{Introduction:}

Spatio-temporal oscillations (electrical and contractile) are fundamental to normal cardiac function but are also a potential source of pathological instability and chaos (1). A stable supply of energy is required to prevent maladaptive emergent phenomena, and mitochondria are well-suited to dynamically adapt to the varying workloads of the organism. Nevertheless, both under physiological conditions (2) or after metabolic stress, mitochondrial oscillations (3), flickers(4),(5), transients(6), or fluctuations(7)(8) have been observed, when parameters such as $\Delta \Psi_{\mathrm{m}}$, flavin or NADH redox potential, $\mathrm{pH}$, or Reactive Oxygen Species (ROS) have been measured. For example, $\Delta \Psi_{m}$, ROS and NADH were shown to oscillate in a self-sustaining manner in adult cardiomyocytes subjected to substrate deprivation (9) or oxidative stress (10) in a frequency range spanning from 1-40 mHz (11). Similarly, local mitochondrial superoxide oscillations ("mitoflashes") in cardiomyocytes had a frequency of $\sim 40 \mathrm{mHz}(12)$. As we have previously reported, $\Delta \Psi_{\mathrm{m}}$ oscillation also reproducibly occurs upon reperfusion after ischemia in neonatal rat ventricular myocyte monolayers (13). Importantly, interventions that suppressed mitochondrial $\Delta \Psi_{\mathrm{m}}$ instability on reperfusion also abrogated cardiac arrhythmias, both in neonatal myocytes (13) and isolated perfused hearts(14),(15). Hence, understanding the mechanism of mitochondrial destabilization during oxidative stress or ischemia/reperfusion (I/R) injury is essential to develop novel therapeutic strategies to prevent cardiac arrhythmias and contractile dysfunction associated with metabolic stress.

Determining the efficacy of interventions targeting spatiotemporal changes in mitochondria requires a robust, unbiased analytical approach, yet there are few reports describing methods for the automated analysis of nonstationary fluctuations observed in image time series. We have previously employed wavelet transform as a tool for characterizing $\Delta \Psi_{\mathrm{m}}$ oscillations and to describe dynamic mitochondrial clustering in adult cardiac myocytes by employing a mesh grid to outline individual mitochondrial clusters (16)(17). Here, we describe a workflow for characterizing spatially distributed $\Delta \Psi_{\mathrm{m}}$ loss and oscillation during $\mathrm{I} / \mathrm{R}$ in terms of time-resolved frequency components, area of mitochondrial clusters, and times of reversible (ischemia) or irreversible (reperfusion) $\Delta \Psi_{\mathrm{m}}$ loss in neonatal cardiac cell monolayers. We apply discrete or continuous wavelet transform methods, followed by feature extraction, to analyze reperfusion-induced unsynchronized $\Delta \Psi_{\mathrm{m}}$ oscillations in neonatal ventricular myocytes. The method accurately identifies key transitions in mitochondrial behavior during I/R and quantifies the principal frequency components of mitochondrial instability and how they evolve over time. Moreover, the method is generalizable to the analysis of spatiotemporal variation of any parameter recorded during image time series. The method provides a workflow to automate microscopy analysis and allows for unbiased, reproducible quantitation of complex nonstationary cellular phenomena.

\section{Methods:}

\section{Neonatal cardiomyocyte isolation and cell culture}

Neonatal mouse cardiac myocytes (NMCMs) were isolated using the MACS cell separation kit (Miltenyi Biotec: Catalog \#130-100-825 and \#130-098-373). Briefly, hearts from 0-2 day old mice were excised, chopped into small pieces and digested using reagents supplied by the kit. A cardiomyocyte-rich cell suspension was obtained by separation of magnetically labelled non-cardiac cells from total cell suspension upon application of a magnetic field. $1 \times 10^{6} \mathrm{NMCMs}$ were plated on fibronectin-coated $(10 \mu \mathrm{g} / \mathrm{ml}) 35 \mathrm{~mm}(\mathrm{D}=20 \mathrm{~mm})$ glass coverslip dishes (NEST ${ }^{\circledR}$ catalog \# 801001) in Medium-199 supplemented with 25mM HEPES, $2 \mu \mathrm{g} / \mathrm{ml}$ Vitamin B12, 50U/ml Pen-strep, 1X non-essential 286 Amino acids and 10\% FBS. The next day, the medium was changed to $2 \%$ FBS medium. Ischemia/Reperfusion experiments and imaging were performed on the 5th- 6 th day of culture.

\section{Inducing Ischemia and Reperfusion and $\Delta \Psi_{m}$ Imaging}

To monitor mitochondrial inner membrane potential $\left(\Delta \Psi_{\mathrm{m}}\right), 50 \mathrm{nM}$ Tetramethylrhodamine methylester (TMRM) was loaded for $30 \mathrm{~min}$ at $37^{\circ} \mathrm{C}$ prior to the start of the experiment and the media was then replaced with fresh 
Tyrode's buffer ( $130 \mathrm{mM} \mathrm{NaCl}, 5 \mathrm{mM} \mathrm{KCl}, 1 \mathrm{mM} \mathrm{MgCl} 2,10 \mathrm{mM} \mathrm{NaHEPES}, 1 \mathrm{mM} \mathrm{CaCl}$ and $5 \mathrm{mM}$ Glucose). Experiment was performed at $37^{\circ} \mathrm{C}$. A typical protocol included a baseline reading for 10 minutes followed by 60 minutes of regional ischemia induced by placing a glass coverslip and followed by 60 minutes of reperfusion upon removal of the coverslip, as previously described in neonatal rat ventricular myocytes (13),(18). During this 130-minute period, images were obtained every $15 \mathrm{sec}$ on a laser-scanning confocal microscope (Olympus FV3000RS). TMRM fluorescence was imaged using a 40X silicone-immersion objective (Olympus UPLSAPO40XS) with 561nm excitation/ 570-620nm emission. Cells were imaged in Galvano scanning mode without averaging. Each image was 16-bit with a size of $318.2 \times 319.2$ microns (512X512 pixels). To minimize laser-induced damage during the long protocol, a neutral density filter of $10 \%$ was applied in the excitation path and the laser intensity was set by the software to $0.06 \%$ power ( $20 \mathrm{~mW} 561 \mathrm{~nm}$ LED laser). At the $15 \mathrm{sec}$ image acquisition interval, only frequencies below $66.67 \mathrm{mHz}$ are resolvable based on Nyquist-Shannon sampling theorem (19).

\section{Image Analysis}

Image series of the time-course of Ischemia/Reperfusion experiments were analyzed using Fiji (https://imagej.net/Fiji/Downloads). A custom-built segmentation-analysis macro was generated to track each cell's $\Delta \Psi_{\mathrm{m}}$ during the in-vitro I/R injury. $\Delta \Psi_{\mathrm{m}}$ response to I/R was analyzed at the cellular level by segmentation analysis (ImageJ). Steps for segmentation analysis included a pre-processing step to align the images in the stack using a 'StackReg' plugin (20). Segmentation of each cell was done by applying a median filter (radius=2) to the first image of the stack and then applying an auto local threshold (Niblack). All particles above the radius of 60 were included in the analysis. TMRM fluorescence intensity for each cell over Ischemia and Reperfusion were obtained. See supplement for macros.

\section{Discrete and Continuous wavelet transform}

Limited information can be obtained through the use of frequency domain methods such as Fourier transform when analyzing complex biological signals that are non-stationary and time varying. Wavelet transform methods, on the other hand, permit resolution of the time of event occurrences and changes in the frequency relationship over time. Signal processing by wavelet transform generates coefficients that represent the best-fit as a selected "mother wavelet" function is scaled and shifted along the source signal(21). There are two kinds of wavelet transforms, Discrete and Continuous wavelet transforms. With Discrete Wavelet Transform (DWT), the signal is decomposed into discrete frequency bands, without overlap of the time-frequency windows of the wavelet function. To detect major transitions that may be hidden in the noise of a physiological signal, the Maximal Overlap Discrete Wavelet Transform (MODWT)(21) can be employed. MODWT decomposes the signal into finer and finer frequency levels. As the level increases, large-scale approximations of the signal are obtained, and lower frequency components of the signal are well-resolved. MODWT of a signal allows for multi-resolution analysis (MRA) that reconstructs the decomposed time series as a sum of several new series that are aligned in time with the original signal. MODWT-MRA effects a zero-phase filtering of the signal. Features are time-aligned, unlike MODWT alone. Continuous Wavelet Transform (CWT) involves transformation of the signal by continuously changing the scaling and shifting factors. Although this introduces some information redundancy, it presents a more detailed, high resolution view of the characteristics of the signal. Coefficients generated by CWT are represented by a scalogram that is a visual representation of the frequency components of the signal as they change over time. In our experiments on cardiomyocytes loaded with the potentiometric fluorophore tetramethylrhodamine methyl ester (TMRM) and subjected to an in vitro I/R protocol, we used MODWT-MRA to identify the timing of the major $\Delta \Psi_{m}$ depolarization during ischemia for each cell. CWT was utilized to analyze the more complex time varying frequency components of the $\Delta \Psi_{\mathrm{m}}$ oscillations observed in individual clusters of mitochondria during reperfusion. The image-processing and wavelet transform workflow, along with feature extraction from the images and scalograms obtained, allowed us to precisely determine the following: 1) the time point of $\Delta \Psi_{\mathrm{m}}$ loss for each cell during Ischemia, 2) the incidence of $\Delta \Psi_{\mathrm{m}}$ oscillation for each mitochondrial cluster 
and its frequency throughout the reperfusion period, 3) whether $\Delta \Psi_{\mathrm{m}}$ stabilized or irreversibly collapsed during reperfusion, and 4) the size distribution of the oscillating mitochondrial clusters.

\section{(i) Identification of transition time-points of inner mitochondrial membrane potentials during Ischemia}

To analyze a time-series of the mitochondrial inner membrane potential, we used MODWT-MRA to identify timelocalized changes in the TMRM signal (using MATLAB's signal processing toolbox). The TMRM signal from each cell during the Ischemic period was transformed with a sym4 wavelet with four levels of decomposition. Lower level decompositions involve higher frequencies and higher-level decompositions involve slower frequencies. For example, Fig. 1 shows a raw TMRM signal from a single cell (A) decomposed into 4 levels using a sym4 wavelet transform (Fig. 1B). Level 1 has the frequency components between $0.033-0.017 \mathrm{~Hz}$, level 2 has $0.017-0.008 \mathrm{~Hz}$, level 3 has 0.008-0.004 and level 4 has 0.004-0.002. All levels of decompositions have associated relative energies. For our purpose of estimating the $\Delta \Psi_{\mathrm{m}}$ depolarization time, we removed all higher frequency components with lower relative energy and reconstructed the signal by retaining the highest relative energy (of more than 99\%) (Fig. 1C). We essentially filter out the 'noise' by this process. With this time-aligned reconstructed signal, we used the MATLAB function 'findchangepoints' to obtain the time point at which the reconstructed signal changed significantly (Fig. 1C). Time point of Ischemia depolarization can thus be automatically determined for several cells (Fig $1 \mathrm{E}$ ).

\section{(ii) Obtaining features and frequency components of $\Delta \Psi_{\mathrm{m}}$ oscillations during reperfusion}

Mitochondria exhibited non-stationary oscillatory behavior throughout reperfusion (Fig 2). We categorized $\Delta \Psi_{\mathrm{m}}$ oscillatory behavior based on our visual observations of 10 experiments. There were five outcomes that were observed based on the oscillatory state of $\Delta \Psi_{\mathrm{m}}$ throughout the reperfusion time period, i.e., (i) $\Delta \Psi_{\mathrm{m}}$ oscillations persisting throughout, (ii) No or very few $\Delta \Psi_{\mathrm{m}}$ oscillations, (iii) $\Delta \Psi_{\mathrm{m}}$ oscillations that stabilized after oscillating initially, (iv) $\Delta \Psi_{m}$ oscillations that occurred, but there was early $\Delta \Psi_{m}$ loss, and (v) No $\Delta \Psi_{m}$ oscillations occurred, and there was early $\Delta \Psi_{\mathrm{m}}$ loss (Fig. 2). We used a continuous wavelet transform (sym8) (in MATLAB's signal processing toolbox), to process the TMRM signal and observed that the signal processing tool readily detected transitions and frequencies depicting the behavior of mitochondrial $\Delta \Psi_{m}$ changes. Figure 2 , right panel, shows the scalograms obtained after performing a wavelet transform of the TMRM signal. We observed that an oscillating cluster has high coefficients concentrated in the scale of $~ 3$ to 10 , corresponding to a frequency of $4.3-45 \mathrm{mHz}$, which does not exist in the scalogram of the non-oscillating cluster or during Ischemia.

This wavelet tool was then applied to detect transitions, frequencies and times associated with these changes automatically for a large number of cells ( $>100$ per experiment) and mitochondrial clusters ( $>400$ per experiment). MATLAB/ FIJ platform was used to perform feature extraction for $\Delta \Psi_{m}$ changes throughout the reperfusion time period (Figure 3). The procedure involved the following steps: (A) image acquisition with a confocal microscope using TMRM to monitor $\Delta \Psi_{m}$ changes; (B) cellular segmentation using custom-made FIJI macros to separate each cell. The same thresholding method was applied to every image to outline each cell in the field of view; (C) By applying the threshold, each cell was separated into an image series; (D) creation of an image Differential Stack of the reperfusion phase of the image series by subtracting the $n^{\text {th }}$ image from the $(n-1)^{\text {th }}$ image. The sum of differentials in this stack could then be used to highlight the mitochondrial clusters that oscillate during the reperfusion period; $(E)$ thresholding the z-projection of this differential image stack to obtain Regions of Interest (ROI) outlining oscillating mitochondrial clusters; (F) application of the ROls to the reperfusion phase to obtain TMRM signals for each cluster through this time period; (G) continuous wavelet transform of the TMRM signal (with a sym 8 wavelet) to generate a coefficient matrix, visualized as a scalogram. The regions on the scalogram with large coefficients indicate where the mother wavelet fits the signal well. The $x$-axis represents the scaled 
time points and $y$-axis represents the scale (scale $\alpha 1 /$ frequency). Usually an oscillating mitochondrion shows high coefficient peaks corresponding to the scale range from 3-10. $\Delta \Psi_{\mathrm{m}}$ can also undergo larger transitions throughout reperfusion and these changes are reflected in the scalograms as high coefficient peaks; $(\mathrm{H})$ importation of the resulting coefficient matrix as a scalogram-image and extraction of predominant frequency features as a function of reperfusion time. $X$ and $Y$ co-ordinates of the outlined maximum coefficients were obtained. The $X$-axis of the scalogram represents the time and the $Y$-axis, the scale (scale $\alpha 1 /$ frequency); (I) Mitochondrial oscillators associated with time are classified into high/low frequency bands. If a mitochondrial cluster oscillates in a particular frequency band at multiple times during reperfusion phase, then, an average of the frequency and the time is obtained. Thus, patterns of oscillatory behavior are obtained. We will henceforth refer to this routine as the MitoWave Analysis.

(See supplement for ImageJ macros and MATLAB codes or on GitHub

https://github.com/dashok1/MitoWave/releases/tag/v1.0.2).

\section{Results:}

\section{Defining oscillatory behavior patterns during Reperfusion}

The behavior of each mitochondrial cluster was plotted into its corresponding frequency band, which varied over the reperfusion time period, represented as violin plots (Fig. 4). Frequencies were categorized as high frequency, ranging from 45 to $4.3 \mathrm{mHz}$ ( 22 seconds to 230 seconds), moderately fast frequencies ranging from $4.3-2.2 \mathrm{mHz}$ ( $\sim 230$ seconds to 450 seconds), slow frequencies ranging from $2.2 \mathrm{mHz}$ to $1.8 \mathrm{mHz}$ ( 450 seconds to $\sim 550$ seconds) and below $1.8 \mathrm{mHz}$. Mitochondrial oscillators typically were present in the $45-4.3 \mathrm{mHz}$ band. We also plotted the time at which there was complete $\Delta \Psi_{\mathrm{m}}$ loss during the reperfusion period. Applying Mito-Wave Analysis on ten in-vitro Ischemia/ Reperfusion experiments, we verified that our visual observations matched the quantitative analysis. In experiments where the mitochondrial oscillations persisted throughout the reperfusion period, high-frequency oscillators appeared at all time periods in the violin plots (Fig 4A) and when mitochondria had few/ no oscillations, the presence of high-frequency oscillators tapered off near 20 minutes of reperfusion (Fig 4B). We also observed, in some experiments, that mitochondrial oscillations occurred in the beginning of reperfusion, but started losing their $\Delta \Psi_{m}$ during mid-late reperfusion, so the high-frequency oscillations tapered off, but shows up in the band where there is $\Delta \Psi_{\mathrm{m}}$ loss (fig $\left.4 \mathrm{C}\right)$. We also observed in some experiments (Fig 4D), mitochondria exhibited few low amplitude or no oscillations at the beginning of reperfusion, so the number of high-frequency oscillators taper off around 20 minutes (similar to the distribution pattern of high frequency oscillators in fig $4 \mathrm{~B}$ ), but they begin to lose their $\Delta \Psi_{\mathrm{m}}$ around 25 minutes of reperfusion. Finally, in some experiments we observed that mitochondria stabilize their $\Delta \Psi_{\mathrm{m}}$ oscillations throughout the reperfusion time period (Fig 4E) where the presence of high-frequency oscillators taper off while $\Delta \Psi_{\mathrm{m}}$ is maintained during reperfusion. We then classified these experiments into the 5 different oscillation categories: Oscillating(4A), NonOscillating(4B), Oscillating with early $\Delta \Psi_{m}$ loss(4C), Oscillating with early $\Delta \Psi_{m}$ stabilization (4D) and Non-Oscillating with early $\Delta \Psi_{m}$ loss (4E).

\section{Predominant frequencies of mitochondrial clusters}

We obtained the predominant frequencies of mitochondrial clusters by considering the first, fast frequency band (8.6- $45 \mathrm{mHz}$ ). If the mitochondrial cluster did not have a frequency in that band, the next frequency band was considered, and so on till the slowest frequency band. This way we could extract the frequencies that most closely represented mitochondrial oscillating frequencies. An average or a weighted average could be used since most mitochondrial clusters also have slow frequency components, but not all mitochondria have fast frequency components. Oscillating clusters have a frequency of $8.73 \pm 4.35 \mathrm{mHz}$ (1081 clusters), Non-Oscillating Clusters have $3.13 \pm 2.61 \mathrm{mHz}$ (732 clusters), Oscillating clusters with early $\Delta \Psi$ loss have $9.56 \pm 3.66 \mathrm{mHz}$ (1402 clusters), Oscillating 
clusters with $\Delta \Psi_{\mathrm{m}}$ stabilization have $8.81 \pm 6.03 \mathrm{mHz}$ (1009 clusters) and Non-Oscillating clusters with Early $\Delta \Psi_{\mathrm{m}}$ loss have $6.82 \pm 4.63 \mathrm{mHz}$ (880 clusters) (figure $5 \mathrm{~A}$ ).

Further, we analyzed the distribution of high frequency oscillators (in the $8.6-45 \mathrm{mHz}$ frequency band) to see how they vary throughout reperfusion time among the different categories. Clusters that didn't have a frequency in this band (of $8.6-45 \mathrm{mHz}$ ) were given a value of 0 . We plotted the percentage of the different categories of oscillating clusters against time (5B). We observed that among the Oscillating category (blue line), $8-12 \%$ of mitochondria exhibited this high-frequency oscillations from 15-40 minutes of reperfusion. This was absent in the Non-Oscillating (orange line), in the Oscillating with early $\Delta \Psi_{m}$ stabilization (Violet line) and the Non-Oscillating with $\Delta \Psi_{m}$ loss (green line) categories. The Oscillating with early $\Delta \Psi_{m}$ loss (pink line) shows $\sim 7-15 \%$ of mitochondria exhibit high frequency only in the early reperfusion phase, till about 25 minutes, after which they do not. Further, we also statistically analyzed the distribution of these high frequency oscillators. A Kolmogorov-Smirnov nonparametric two sample test (kstest2 on MATLAB) was performed to test the null hypothesis that distribution of various oscillation behaviors were not different during the reperfusion time period. KS-test show significant differences between the different categories, comparing Oscillating and Non-Oscillating clusters, Oscillating and Oscillating with early $\Delta \Psi_{\mathrm{m}}$ loss, Oscillating and $\Delta \Psi_{\mathrm{m}}$ stabilizing clusters, and Oscillating and Non-Oscillating with early $\Delta \Psi$ m loss $(p<0.0001)$. Thus, we quantitatively confirm our visual observations that the distribution of oscillating mitochondrial clusters that change dynamically over time are different between different categories of oscillating experiments.

\section{Frequency and mitochondrial cluster size are negatively correlated}

We observed that in experiments where there were no/ few oscillations, mitochondrial clusters seem larger than in experiments where mitochondria had persistent oscillations. Previous reports in adult cardiac myocytes also showed that larger clusters have slower oscillations (11). Therefore, we wanted to check if this was true in Neonatal Cardiac myocytes as well. The Mito-Wave analysis of NMVMs subjected to I/R agreed with our visual observations. Oscillating mitochondria had the lowest area of $49.3 \mu \mathrm{m}^{2}$ vs a larger area of $65.92 \mu \mathrm{m}^{2}$ for nonoscillating mitochondria (Fig 6A). We performed non-parametric correlation coefficient analysis to understand the relationship between the size of mitochondrial clusters and its frequency. We found that there is a negative correlation between oscillating frequency and the size of the mitochondrial cluster, with a correlation coefficient of $r=-0.58$ (Fig. 6B). Mitochondrial cluster size decreased by $\sim 4.56 \mu \mathrm{m}^{2}$ for every millihertz increase. This suggests that if mitochondria are organized in larger clusters, they undergo slower oscillations and may eventually stabilize $\Delta \Psi_{\mathrm{m}}$ and be protected against $\Delta \Psi_{\mathrm{m}}$ loss during reperfusion after Ischemia.

\section{Time taken for $\Delta \Psi \mathrm{m}$ loss during Ischemia and Reperfusion}

Time to $\Delta \Psi_{m}$ loss (reversible during Ischemia and irreversible during reperfusion) is an important indicator for mitochondrial resistance to instability during reperfusion after ischemia. It helps to understand if interventions to prevent mitochondrial instability and hence reperfusion injury are effective. We quantified the time to $\Delta \Psi_{m}$ loss during Ischemia per cell (7A). The Oscillating category had a mean of $43.52 \pm 5.87$ minutes to $\Delta \Psi_{m}$ loss (i), NonOscillating took $46.36 \pm 9.17$ minutes(ii), Oscillating with early $\Delta \Psi_{\mathrm{m}}$ loss took $35.62 \pm 9.25$ minutes(iii), Oscillating with $\Delta \Psi_{\mathrm{m}}$ stabilization took $52.84 \pm 11.17$ minutes (iv) and Non-Oscillating clusters with Early $\Delta \Psi_{\mathrm{m}}$ loss took $30.46 \pm 7.81$ minutes $(\mathrm{v})$. We also quantified the time to $\Delta \Psi_{\mathrm{m}}$ loss per mitochondrion during reperfusion (7B). We plotted the time against the percentage of mitochondria. Oscillating clusters take $58.71 \pm 4.75$ minutes to lose $\Delta \Psi_{\mathrm{m}}$; Non-Oscillating clusters did not lose their $\Delta \Psi_{\mathrm{m}}$ till the end of reperfusion at 60.25 minutes; Oscillating clusters with early $\Delta \Psi \mathrm{m}$ loss take $45.8 \pm 11.05$ minutes; Oscillating clusters with $\Delta \Psi \mathrm{m}$ stabilization take $59.66 \pm 3.96$ minutes and Non-Oscillating clusters with early $\Delta \Psi_{\mathrm{m}}$ loss take $53.38 \pm 10.99$ minutes. 


\section{Correlation between Ischemic depolarization time point and $\Delta \Psi_{\mathrm{m}}$ oscillation frequency}

We wanted to understand if there was any link between mitochondrial recovery during reperfusion and the time to $\Delta \Psi_{\mathrm{m}}$ loss during Ischemia. We compared the empirical cumulative distribution functions between different oscillation categories during Ischemia (8A) and reperfusion (8B). We found that late $\Delta \Psi_{\mathrm{m}}$ loss during Ischemia correlated with mitochondrial $\Delta \Psi_{\mathrm{m}}$ stabilization during reperfusion.

\section{Discussion:}

Over the course of ischemia-reperfusion, the mitochondrial networks of cultured neonatal mouse cardiomyocytes displayed complex spatiotemporal patterns, including bistability and time-varying oscillatory behavior, presenting significant challenges to analysis. The present work combined image segmentation with the versatility of wavelet transforms to quantify key transitions associated with the pathophysiology of $\mathrm{I} / \mathrm{R}$ injury in an unbiased manner. Essential information could be captured in a semi-automated workflow, including the time-to-mitochondrial depolarization during ischemia, frequency of $\Delta \Psi_{m}$ oscillation of individual mitochondrial clusters upon reperfusion, and time to catastrophic loss of $\Delta \Psi_{m}$ with prolonged reperfusion. Subsequent data reduction permits one to make statistical comparisons between different experiments to determine if a given treatment or intervention has significant effect on mitochondrial function (Fig 3).

We have previously reported that adult cardiomyocytes subjected to metabolic or oxidative stress undergo spontaneous oscillations in $\Delta \Psi_{\mathrm{m}}$ that occur either in small clusters or are synchronized across the whole cell (11). Cell wide $\Delta \Psi_{\mathrm{m}}$ synchronization is observed after a critical number of mitochondria in the network show oxidative stress, a phenomenon we termed "mitochondrial criticality"(22). Synchronization of mitochondria in the organized array of the adult myocyte depends on ROS-dependent neighbor-neighbor interactions between organelles, with long range cluster interactions following the behavior of a percolation lattice(23). In neonatal myocytes, the mitochondrial network is less ordered and reperfusion-induced oscillations are less likely to be synchronized throughout the entire network (13), consistent with a short effective diffusion distance for ROSinduced ROS release(10). In contrast, when the system is forced by a uniform environmental stress, such as ischemia, mitochondrial network depolarization occurs on a cell-by-cell basis, likely determined by the anaerobic ATP-generating capacity and glycogen store of the individual cells. The average time to ischemic $\Delta \Psi_{\mathrm{m}}$ depolarization for a given coverslip was compared to the oscillatory behavior of mitochondrial clusters on reperfusion (Fig. $7 \&$ 8). Interestingly, early $\Delta \Psi_{m}$ loss during ischemia correlated with early $\Delta \Psi_{m}$ loss during reperfusion; however, this was equally true for both oscillating and non-oscillating clusters, suggesting that there is no specific protective advantage of the oscillatory behavior. In fact, there was a trend towards earlier depolarization during reperfusion for oscillating versus non-oscillating mitochondrial clusters. At least concerning mitochondrial recovery after reperfusion, these findings argue against the idea that oscillations in metabolism might preserve a higher average ATP/ADP ratio while decreasing free energy dissipation compared to steady state operation (24). Instead, mitochondrial $\Delta \Psi_{m}$ oscillation could simply be an inevitable consequence of the nonlinear control properties of the nonlinear bioenergetic system. In addition, late $\Delta \Psi_{\mathrm{m}}$ loss during ischemia correlated with $\Delta \Psi_{\mathrm{m}}$ stabilization after oscillation on reperfusion. Together these data indicate that mitochondrial energetic recovery strongly depends on resistance to initial ischemic depolarization, consistent with data from intact perfused hearts(25).

The present findings show that in NMVMs subjected to I/R, $\Delta \Psi_{m}$ oscillation frequency is inversely correlated with cluster size (Fig. 6). This is in agreement with the negative correlation obtained by wavelet transform analysis of adult myocytes under oxidative stress, with large mitochondrial clusters showing slow $\Delta \Psi_{\mathrm{m}}$ oscillations that could span the entire cell with a stereotypical frequency of $1-10 \mathrm{mHz}(11)$. Synchronization of a network of dynamically coupled oscillators spanning a broad frequency range to a single dominant frequency is common to physical, chemical and biological systems. The lack of synchronization in NMVMs and the broader frequency distribution 
(Fig. 5) may be the result of the more disorganized arrangement of mitochondria in neonatal myocytes or weaker coupling between mitochondria in the immature cells.

The method described here provides a way to uncover and quantify different mitochondrial responses to I/R stress that might otherwise be overlooked if one were to only examine the average behavior of a monolayer, of individual cells, or at single time points during a protocol (e.g., measuring lactate dehydrogenase release as an index of damage after reperfusion). A current limitation of the method is that it would be affected by significant movement of the objects being analyzed in the optical field, which was minimal in our experiments. In the future, it might be possible to further develop the approach by incorporating object tracking methods. Nevertheless, the approach is applicable to any spatially-distributed system of time varying oscillatory signals. Unlike Fourier transform analysis, the underlying oscillator frequencies and phases do not have to be time invariant and the method is largely immune to changes in signal offset (such as photobleaching) or background artifacts. This novel approach, which standardizes the quantitative analysis of complex biological signals, opens the door to in depth screening of the genes, proteins and mechanisms underlying metabolic recovery after ischemia-reperfusion.

\section{Acknowledgements:}

We are grateful to Dr. Amitabh Basu, for discussions on statistical analysis and MATLAB coding

This work was supported by NIH grants R01HL137259, R01HL134821 (BO'R) and F31HL134198 (DA).

\section{Author Contributions:}

D.A. performed experiments, performed analysis, wrote codes for analysis and wrote the paper.

B.O'R conceived the idea and wrote the paper

\section{Supplemental information:}

1. MitoWave Analysis Routine ImageJ and MATLAB codes (also on GitHub https://github.com/dashok1/MitoWave/releases/tag/v1.0.2 and zenodo https://doi.org/10.5281/zenodo.3820382)

2. In-vitro Ischemia/Reperfusion experiment movie showing mitochondria Oscillating throughout reperfusion: Oscillating Category

3. In-vitro Ischemia/Reperfusion experiment movie showing mitochondria Not Oscillating throughout reperfusion: Non-Oscillating Category

4. In-vitro Ischemia/Reperfusion experiment movie showing mitochondria Oscillating, then losing $\Delta \Psi_{\mathrm{m}}$ during reperfusion: Oscillating with early $\Delta \Psi_{\mathrm{m}}$ loss Category

5. In-vitro Ischemia/Reperfusion experiment movie showing mitochondria Oscillating, then stabilizing during reperfusion: Oscillating with early $\Delta \Psi_{\mathrm{m}}$ stabilization Category

6. In-vitro Ischemia/Reperfusion experiment movie showing mitochondria Not Oscillating and exhibiting early $\Delta \Psi_{\mathrm{m}}$ loss during reperfusion: Non-Oscillating with early $\Delta \Psi_{\mathrm{m}}$ loss Category 


\section{References:}

1. Garfinkel, A., M.L. Spano, W.L. Ditto, and J.N. Weiss. 1992. Controlling Cardiac Chaos. Science. 257:1230-1235.

2. Mironov, S.L., and D.W. Richter. 2001. Oscillations and hypoxic changes of mitochondrial variables in neurons of the brainstem respiratory centre of mice. J. Physiol. 533:227-236.

3. Siemens, A., R. Walter, L.H. Liaw, and M.W. Berns. 1982. Laser-stimulated fluorescence of submicrometer regions within single mitochondria of rhodamine-treated myocardial cells in culture. Proc. Natl. Acad. Sci. U. S. A. 79:466-470.

4. Duchen, M.R., A. Leyssens, and M. Crompton. 1998. Transient Mitochondrial Depolarizations Reflect Focal Sarcoplasmic Reticular Calcium Release in Single Rat Cardiomyocytes. J. Cell Biol. 142:975988.

5. Nivala, M., P. Korge, M. Nivala, J.N. Weiss, and Z. Qu. 2011. Linking Flickering to Waves and WholeCell Oscillations in a Mitochondrial Network Model. Biophys. J. 101:2102-2111.

6. Romashko, D.N., E. Marban, and B. O’Rourke. 1998. Subcellular metabolic transients and mitochondrial redox waves in heart cells. Proc. Natl. Acad. Sci. 95:1618-1623.

7. Buckman, J.F., and I.J. Reynolds. 2001. Spontaneous Changes in Mitochondrial Membrane Potential in Cultured Neurons. J. Neurosci. 21:5054-5065.

8. Vergun, O., and I.J. Reynolds. 2004. Fluctuations in Mitochondrial Membrane Potential in Single Isolated Brain Mitochondria: Modulation by Adenine Nucleotides and Ca2+. Biophys. J. 87:35853593.

9. O’Rourke, B., B.M. Ramza, and E. Marban. 1994. Oscillations of membrane current and excitability driven by metabolic oscillations in heart cells. Science. 265:962-966.

10. Aon, M.A., S. Cortassa, E. Marbán, and B. O’Rourke. 2003. Synchronized Whole Cell Oscillations in Mitochondrial Metabolism Triggered by a Local Release of Reactive Oxygen Species in Cardiac Myocytes. J. Biol. Chem. 278:44735-44744.

11. Kurz, F.T., M.A. Aon, B. O’Rourke, and A.A. Armoundas. 2010. Spatio-temporal oscillations of individual mitochondria in cardiac myocytes reveal modulation of synchronized mitochondrial clusters. Proc. Natl. Acad. Sci. 107:14315-14320.

12. Wang, W., H. Fang, L. Groom, A. Cheng, W. Zhang, J. Liu, X. Wang, K. Li, P. Han, M. Zheng, J. Yin, W. Wang, M.P. Mattson, J.P.Y. Kao, E.G. Lakatta, S.-S. Sheu, K. Ouyang, J. Chen, R.T. Dirksen, and H. Cheng. 2008. Superoxide Flashes in Single Mitochondria. Cell. 134:279-290.

13. Solhjoo, S., and B. O'Rourke. 2015. Mitochondrial instability during regional ischemia-reperfusion underlies arrhythmias in monolayers of cardiomyocytes. J. Mol. Cell. Cardiol. 78:90-99. 
14. Akar, F.G., M.A. Aon, G.F. Tomaselli, and B. O'Rourke. 2005. The mitochondrial origin of postischemic arrhythmias. J. Clin. Invest. 115:3527-3535.

15. Zhou, L., S. Solhjoo, B. Millare, G. Plank, M.R. Abraham, S. Cortassa, N. Trayanova, and B. O’Rourke. 2014. Effects of Regional Mitochondrial Depolarization on Electrical Propagation: Implications for Arrhythmogenesis. Circ. Arrhythm. Electrophysiol. 7:143-151.

16. Kurz, F.T., M.A. Aon, B. O’Rourke, and A.A. Armoundas. 2010. Wavelet analysis reveals heterogeneous time-dependent oscillations of individual mitochondria. Am. J. Physiol. - Heart Circ. Physiol. 299:H1736-H1740.

17. Kurz, F.T., M.A. Aon, B. O’Rourke, and A.A. Armoundas. 2014. Cardiac mitochondria exhibit dynamic functional clustering. Front. Physiol. 5.

18. Pitts, K.R., and C.F. Toombs. 2004. Coverslip hypoxia: a novel method for studying cardiac myocyte hypoxia and ischemia in vitro. Am. J. Physiol.-Heart Circ. Physiol. 287:H1801-H1812.

19. Marks, R. 1991. Introduction to Shannon Sampling and Interpolation Theory. New York: SpringerVerlag.

20. Thévenaz, P., U.E. Ruttimann, and M. Unser. 1998. A Pyramid Approach to Subpixel Registration Based on Intensity. IEEE Trans. Image Process. 7:27-41.

21. Percival, D.B. 2008. Analysis of Geophysical Time Series Using Discrete Wavelet Transforms: An Overview. In: Donner RV, SM Barbosa, editors. Nonlinear Time Series Analysis in the Geosciences. Berlin, Heidelberg: Springer Berlin Heidelberg. pp. 61-79.

22. Aon, M.A., S. Cortassa, F.G. Akar, and B. O’Rourke. 2006. Mitochondrial criticality: A new concept at the turning point of life or death. Biochim. Biophys. Acta. 1762:232-240.

23. Aon, M.A., S. Cortassa, and B. O’Rourke. 2004. Percolation and criticality in a mitochondrial network. Proc. Natl. Acad. Sci. U. S. A. 101:4447-4452.

24. Termonia, Y., and J. Ross. 1981. Oscillations and control features in glycolysis: numerical analysis of a comprehensive model. Proc. Natl. Acad. Sci. U. S. A. 78:2952-2956.

25. Lyon, A.R., P.J. Joudrey, D. Jin, R.D. Nass, M.A. Aon, B. O’Rourke, and F.G. Akar. 2010. Optical imaging of mitochondrial function uncovers actively propagating waves of mitochondrial membrane potential collapse across intact heart. J. Mol. Cell. Cardiol. 49:565-575. 


\section{was not certified by peer review) is the author/funder, who has granted bioRxiv a license to display the preprint in perpetuity. It is made}

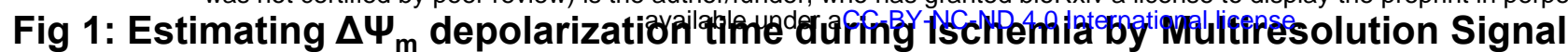
Analysis

(A) TMRM signal from a single cell during $I / R$

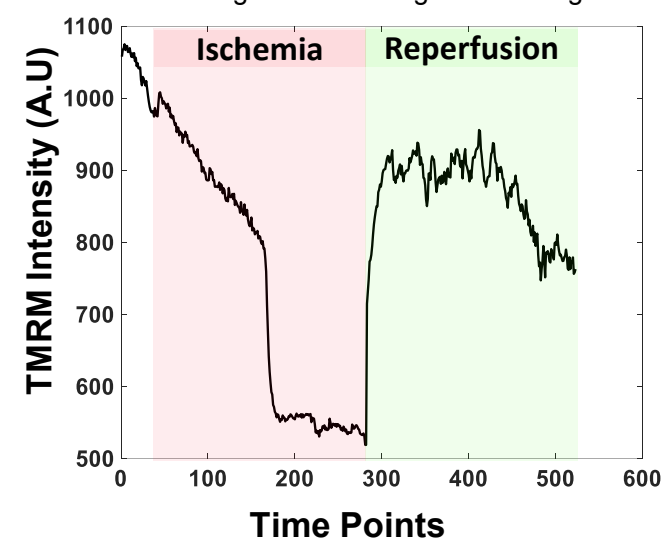

(D)

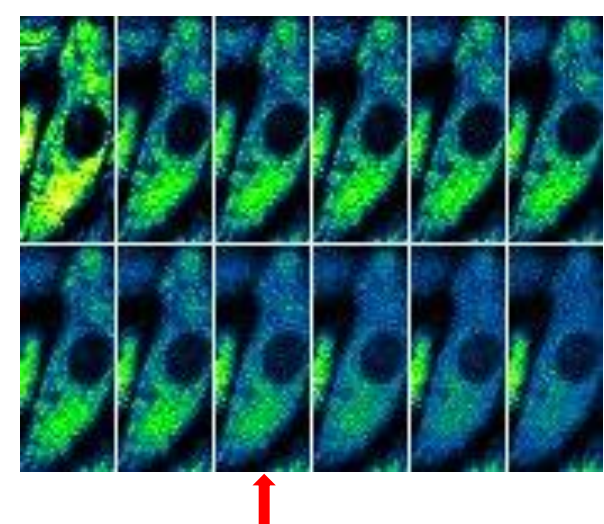

(B)

Decomposition of Signal

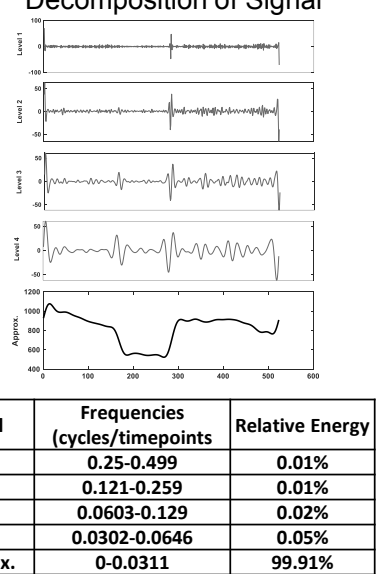

(C) Reconstructed Signal

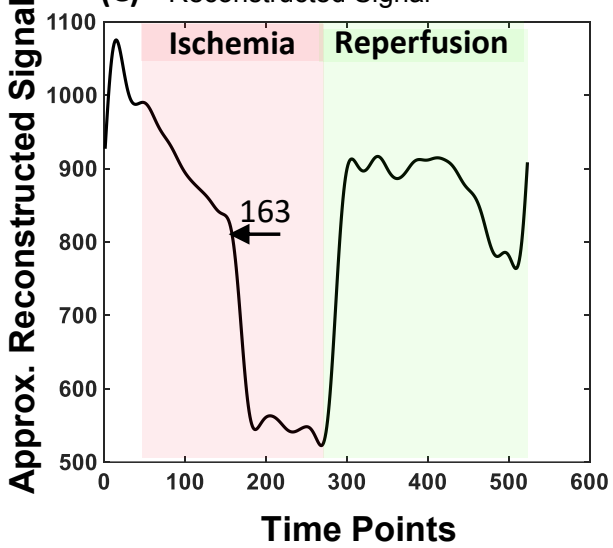

(E)

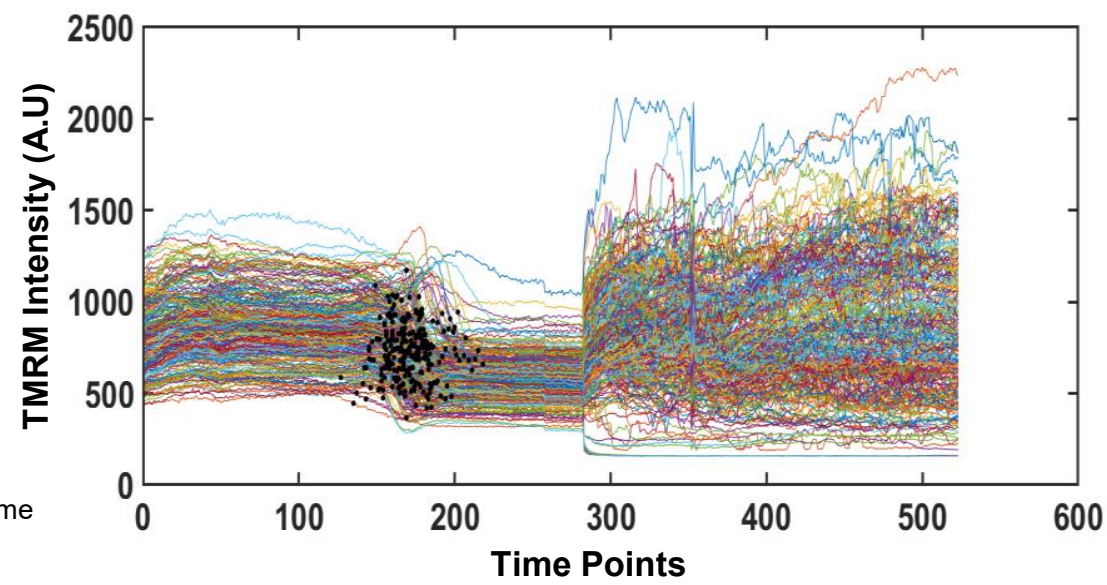

Figure 1: Identification of Ischemic depolarization time. Raw TMRM signals during Ischemic period (A) are decomposed using Maximal Overlap Discrete Wavelet Transform (B) and reconstructed by retaining the signal with the highest relative energy (C). MATLAB's 'findchangepoints' function identifies the time point at which the signal changed significantly during Ischemia. Here, it is at time point 163. D) Example of a cell with the first image at baseline and subsequent images in the last phases of depolarization. Images are 15 seconds apart. TMRM intensity is abruptly diminished at time point 163. E) Example of Ischemia/ Reperfusion experiment with $>100$ cells where the black dots represent Ischemic depolarization time points. 
Figure 3: Workflow for characterizing $\Delta \Psi \mathrm{m}$ oscillatory behavior during reperfusion: MitoWave

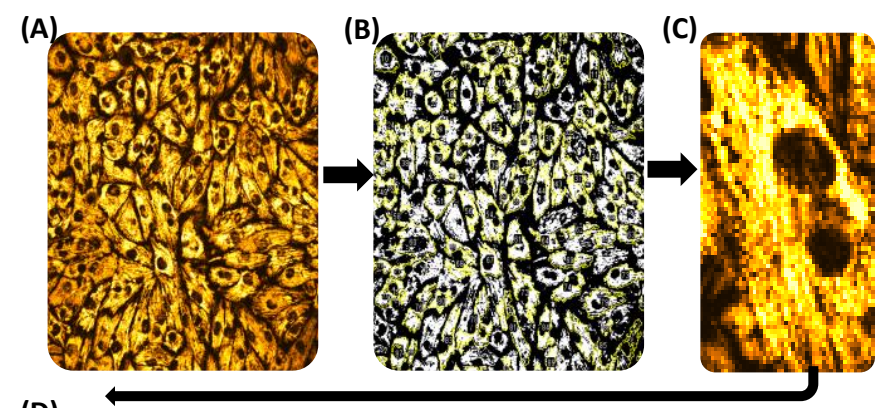

Image Acquisition and cellular segmentation

(D)

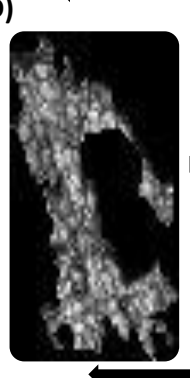

(G)

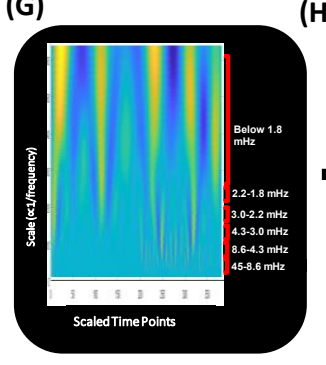

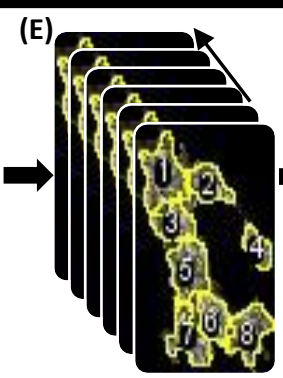

(H)

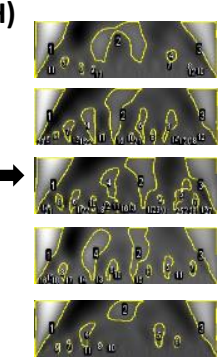

(F)

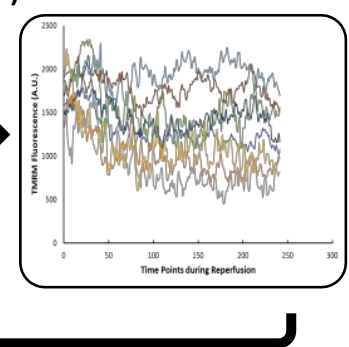

(I)

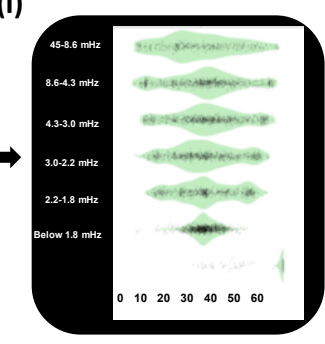

Mito-cluster segmentation to obtain $\Delta \Psi \mathrm{m}$ changes through reperfusion
Continuous Wavelet Transform of TMRM signals from each mito-cluster during reperfusion and feature extraction

Figure 3: Schematic of Mito-wave analysis for $\Delta \Psi_{\mathrm{m}}$ feature extraction. It involves the following steps: A) Image Acquisition with a Confocal microscope using TMRM to monitor $\Delta \Psi \mathrm{m}$ changes, B-C) Cellular Segmentation using custom-made FIJI macros to separate each cell, D) Differential stack Zprojection image for each cell is used to identify mitochondrial clusters that oscillate (MATLAB/FIJI Routine), E-F) TMRM fluorescence time course for each cluster is obtained, G) Scalograms are generated by continuous wavelet transform of the TMRM signal, $H$ ) Features and frequency components are extracted from the scalograms, and I) Mitochondrial oscillators are classified into high/low frequency bands to obtain patterns of oscillatory behavior as a function of reperfusion time. 
was ro

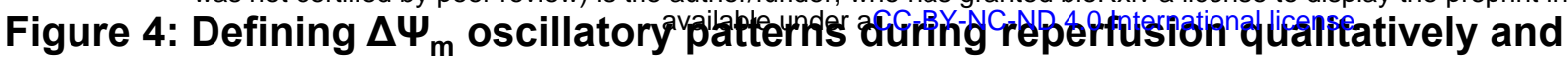
quantitatively.

\begin{tabular}{|c|l|}
\hline Table 1 & Visual Observations during reperfusion \\
\hline $\mathbf{1}$ & Oscillating mitochondrial clusters \\
\hline $\mathbf{2}$ & No Oscillations during reperfusion \\
\hline $\mathbf{3}$ & Oscillations with early $\Delta \Psi \mathrm{m}$ loss \\
\hline $\mathbf{4}$ & Yes Oscillations, then stabilization \\
\hline $\mathbf{5}$ & No fast Oscillations, but show early $\Delta \Psi \mathrm{m}$ loss \\
\hline
\end{tabular}

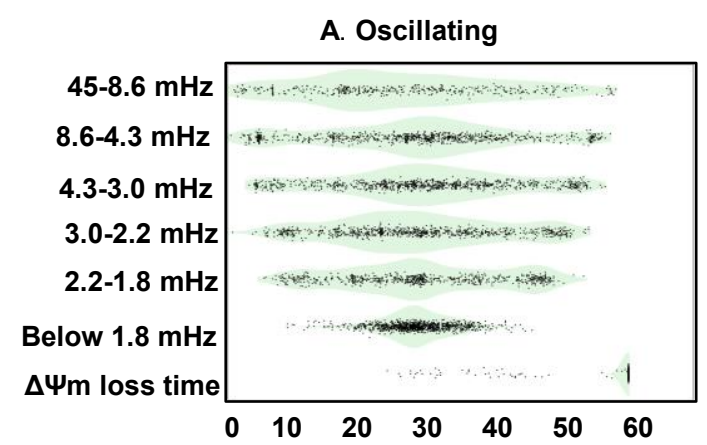

C. Oscillating, with early $\Delta \Psi \mathrm{m}$ loss

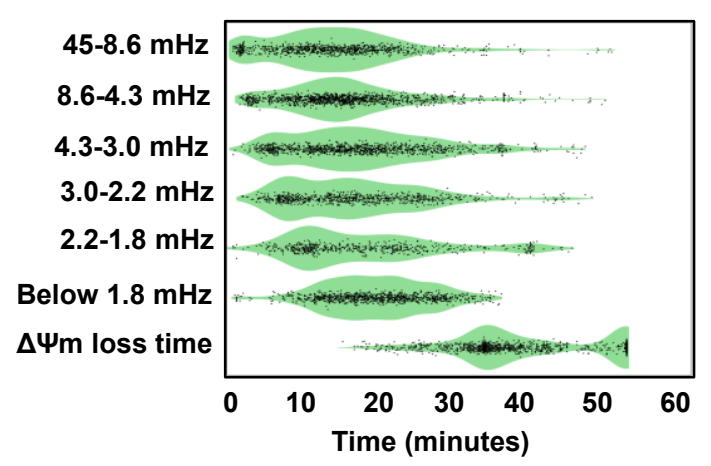

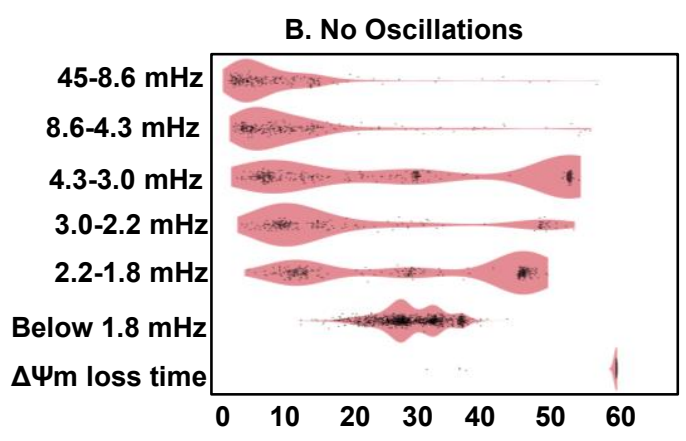

D. Oscillating, with $\Delta \Psi \mathrm{m}$ stabilization

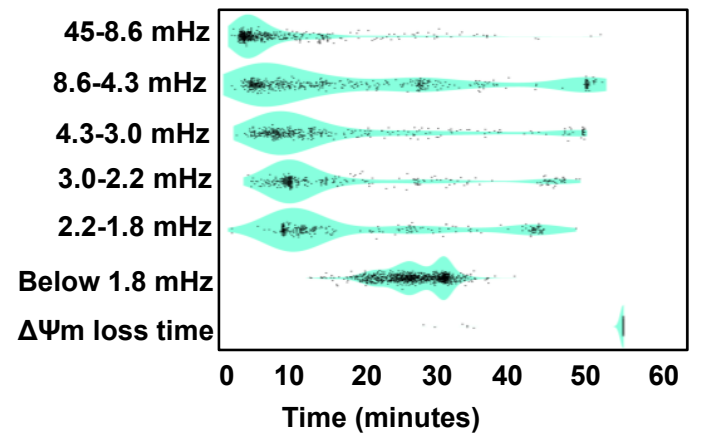

\section{E. No Oscillations, with $\Delta \Psi \mathrm{m}$ loss}

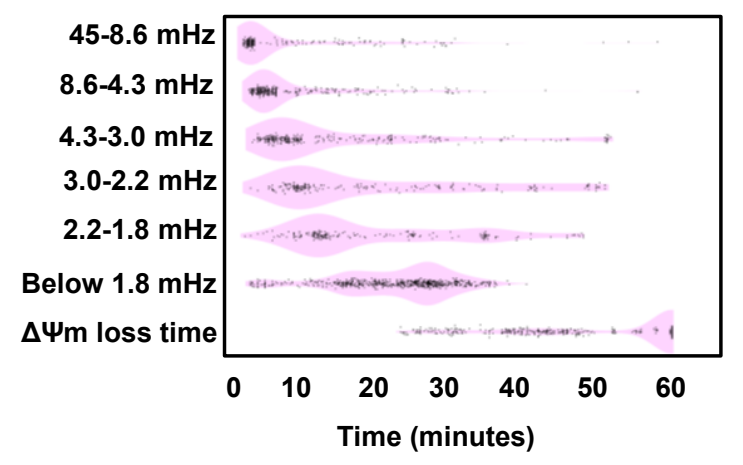

Figure 4: Defining $\Delta \boldsymbol{\Psi}_{\mathrm{m}}$ Oscillatory patterns during reperfusion qualitatively and quantitatively:

Visual observations of the Ischemia/Reperfusion image stack can qualitatively classify oscillatory behavior patterns of mitochondrial clusters during reperfusion. We classified oscillatory patterns from 10 experiments into 5 groups: Oscillating, Not Oscillating, Oscillating with early $\Delta \Psi_{\mathrm{m}}$ loss, Oscillating with $\Delta \Psi_{\mathrm{m}}$ stabilization, and Non-Oscillating clusters with Early $\Delta \Psi_{\mathrm{m}}$ loss (Table 1). By subjecting the TMRM signal from each mitochondrial cluster to MitoWave Analysis, we characterize oscillatory behavior quantitatively with violin plots (Fig. 4A-E). Each dot represents a mitochondrial cluster oscillating at a certain frequency corresponding to a certain time point. Visual observations (Table 1) are corroborated by results from the quantitative MitoWave analysis routine (fig $4 \mathrm{~A}-\mathrm{E}$ ). We see that a mitochondrial cluster can change its oscillatory pattern throughout the reperfusion period, i.e., its frequency may change from one frequency band to another. Y-axis shows six frequency bands, as well as the time at which a mitochondrial cluster completely loses $\Delta \Psi \mathrm{m}$ during reperfusion. X-axis represents the time of reperfusion. 
Fiare reperfusion.

A. Frequency of Mitochondrial Clusters

B. Percentage of fast oscillating mitochondrial clusters

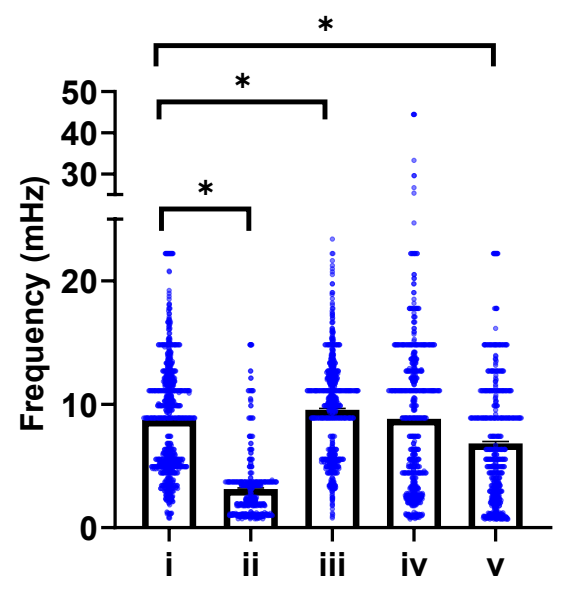
existing in a 4-minute time intervals during reperfusion

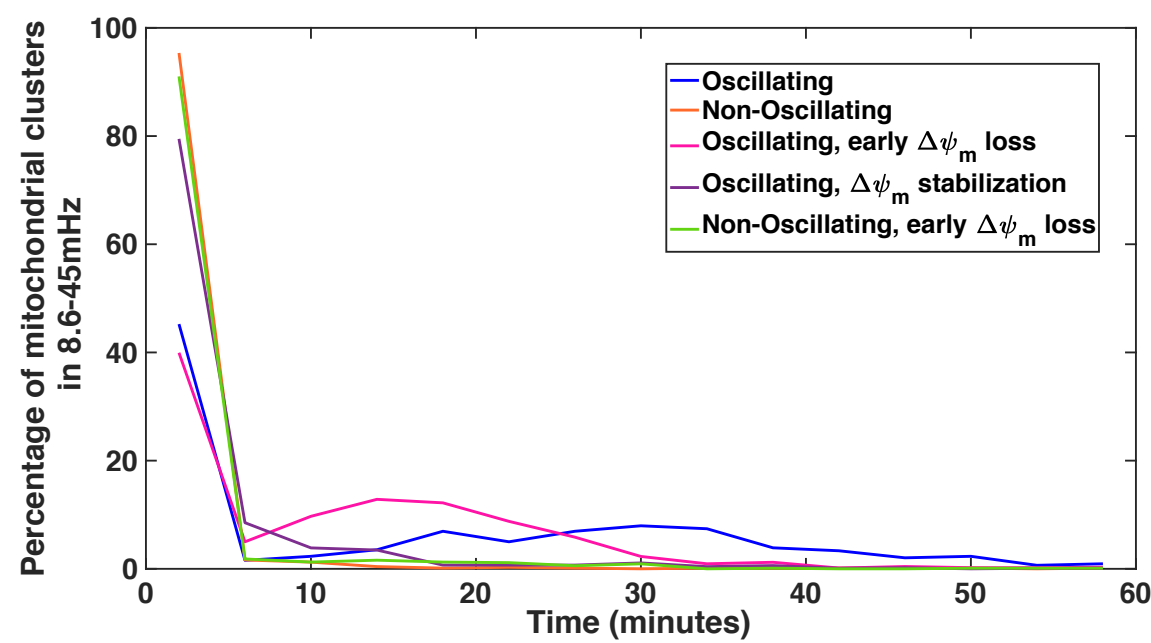

Figure 5: The predominant frequencies exhibited by mitochondrial clusters fell within the 8.6 to $45 \mathrm{mHz}$ band. A) The mean predominant frequency \pm SEM for i) Oscillating clusters, $8.73 \pm 4.35 \mathrm{mHz}(1081$ clusters); ii) Non-Oscillating Clusters, $3.13 \pm 2.61 \mathrm{mHz}$ (732 clusters); iii) Oscillating cluster with early $\Delta \Psi$ loss, $9.56 \pm 3.66 \mathrm{mHz}$ (1402 clusters); iv) Oscillating cluster with $\Delta \Psi$ stabilization, $8.81 \pm 6.03 \mathrm{mHz}$ (1009 clusters); and v) Non-Oscillating clusters with early $\Delta \Psi \mathrm{m}$ loss, $6.82 \pm 4.63 \mathrm{mHz}$ ( 880 clusters). One-way ANOVA was performed to determine statistical significance, $* p<0.0001$. B) Percentage of mitochondrial clusters oscillating in the $8.6-45 \mathrm{mHz}$ frequency band binned at 4-minute intervals during the reperfusion period. 


\section{A. Area of Mitochondrial Clusters}

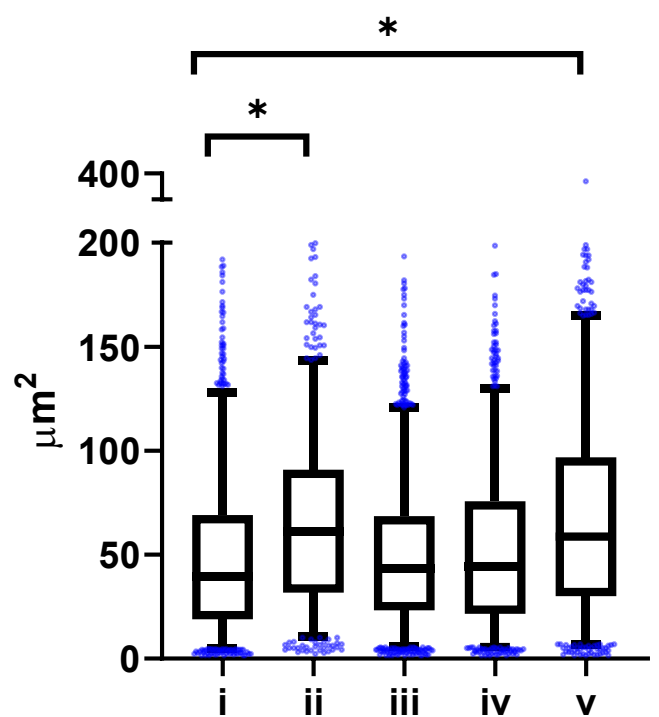

\section{B. Oscillating Clusters}

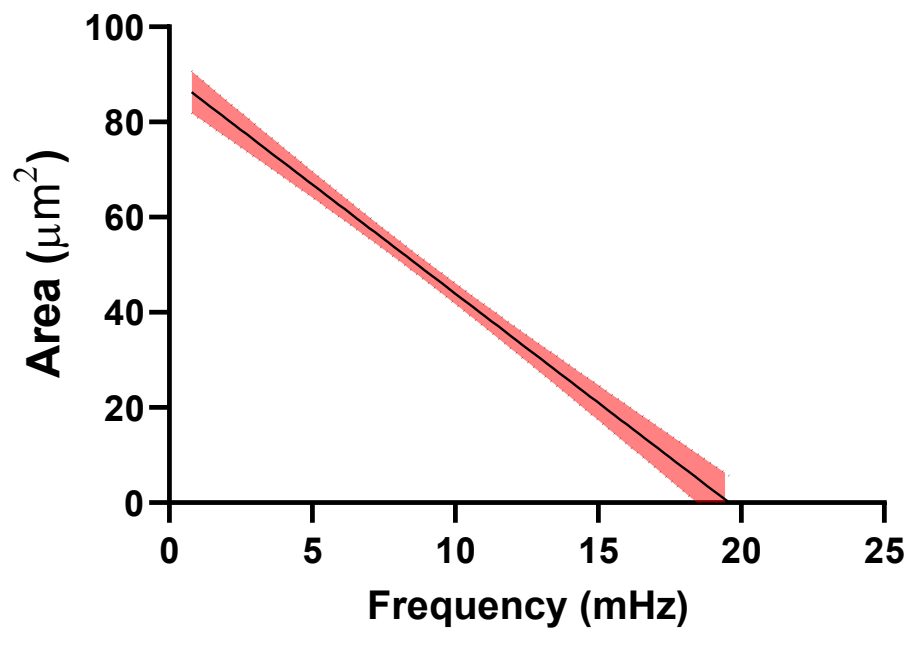

Figure 6: A) Mitochondrial Cluster size and Frequency relationship. Areas of mitochondrial clusters were compared for clusters exhibiting different oscillatory behaviors (across several experiments). (i) Oscillating clusters had an area of $49.78 \pm 40.64 \mu \mathrm{m}^{2}$ (1081 clusters); (ii) Non-Oscillating Clusters, $65.97 \pm 42.07 \mu \mathrm{m}^{2}$ (732 clusters); (iii) Oscillating cluster with early $\Delta \Psi$ loss, $49.65 \pm 34.35 \mu \mathrm{m}^{2}$ (1402 clusters); (iv) Oscillating cluster with $\Delta \Psi$ stabilization, $53.15 \pm 39.38 \mu \mathrm{m}^{2}$ (1009 clusters); and (v) Non-Oscillating clusters with Early $\Delta \Psi \mathrm{m}$ loss, $67.92 \pm 49.12 \mu \mathrm{m}^{2}$ (880 clusters). One-way ANOVA was performed to determine statistical significance, $* p<0.0001$. B) Frequency and cluster size show an inverse relationship. In Oscillating clusters, the area of the cluster decreases by $\sim 4.56 \mu \mathrm{m}^{2}$ for every millihertz increase. $95 \%$ confidence intervals are plotted (red) with linear regression line (black). 


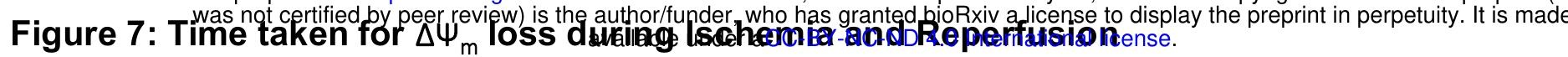

\section{A. Ischemia $\Delta \Psi_{m}$ depolarization time}

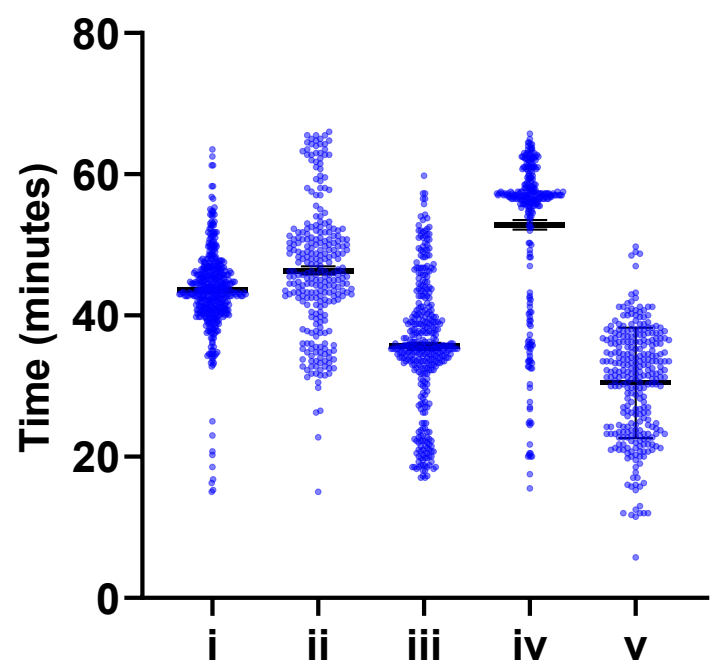

B. Percent of mitochondrial clusters exhibiting irreversible $\Delta \Psi \mathrm{m}$ loss during reperfusion phase

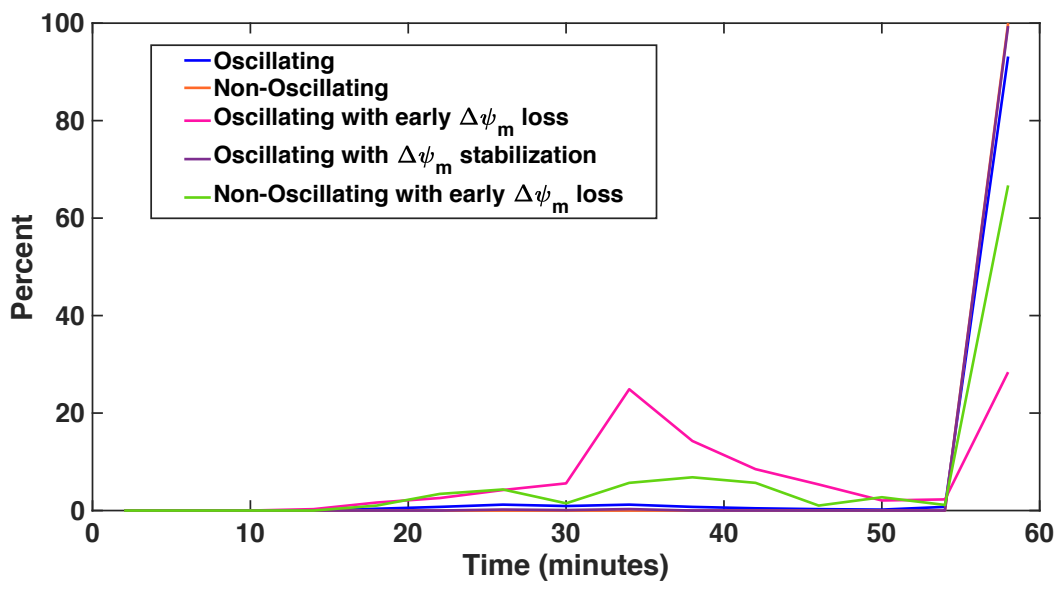

Figure 7 : Time taken for $\Delta \Psi_{m}$ loss during Ischemia and Reperfusion

A) Time taken for $\Delta \Psi_{m}$ loss during Ischemia versus the ensuing oscillatory behavior on reperfusion. (i) Oscillating clusters maintained $\Delta \Psi_{\mathrm{m}}$ until $43.52 \pm 5.87$ minutes; (ii) Non-Oscillating clusters, $46.36 \pm 9.17$ minutes; (iii) Oscillating with early $\Delta \Psi_{m}$ loss, $35.62 \pm 9.25$ minutes (iv) Oscillating with early $\Delta \Psi_{m}$ stabilization, 52.84 \pm 11.17 minutes and (v) NonOscillating clusters with Early $\Delta \Psi_{\mathrm{m}}$ loss, $30.46 \pm 7.81$ minutes.

B) Percentage of mitochondrial clusters exhibiting irreversible $\Delta \Psi_{\mathrm{m}}$ loss during reperfusion. Oscillating clusters lost $\Delta \Psi_{\mathrm{m}}$ on average at 58.71 \pm .75 minutes of reperfusion; Non-Oscillating clusters maintained stable $\Delta \Psi_{\mathrm{m}}$ to the end of 60.25 minutes of reperfusion; Oscillating clusters with early $\Delta \Psi_{m}$ loss depolarized at $45.8 \pm 11.05$ minutes; Oscillating clusters with $\Delta \Psi_{\mathrm{m}}$ stabilization lasted $59.66 \pm 3.96$ minutes, and Non-Oscillating clusters with early $\Delta \Psi_{\mathrm{m}}$ loss depolarized at $53.38 \pm 10.99$ minutes 
Figure 8: Relationship between Ischemia $\Delta \Psi_{m}$ depolarization time with Oscillatory behavior during Reperfusion

A. ECDF: $\Delta \Psi \mathrm{m}$ loss during Ischemia

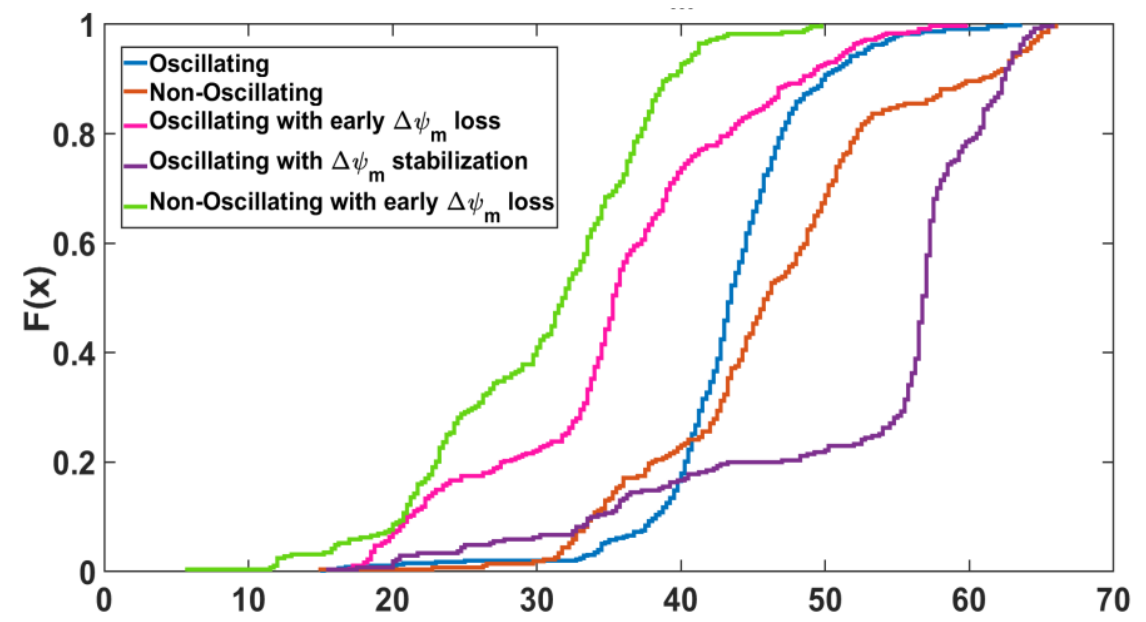

B. ECDFs: $\Delta \Psi \mathrm{m}$ loss during Reperfusion

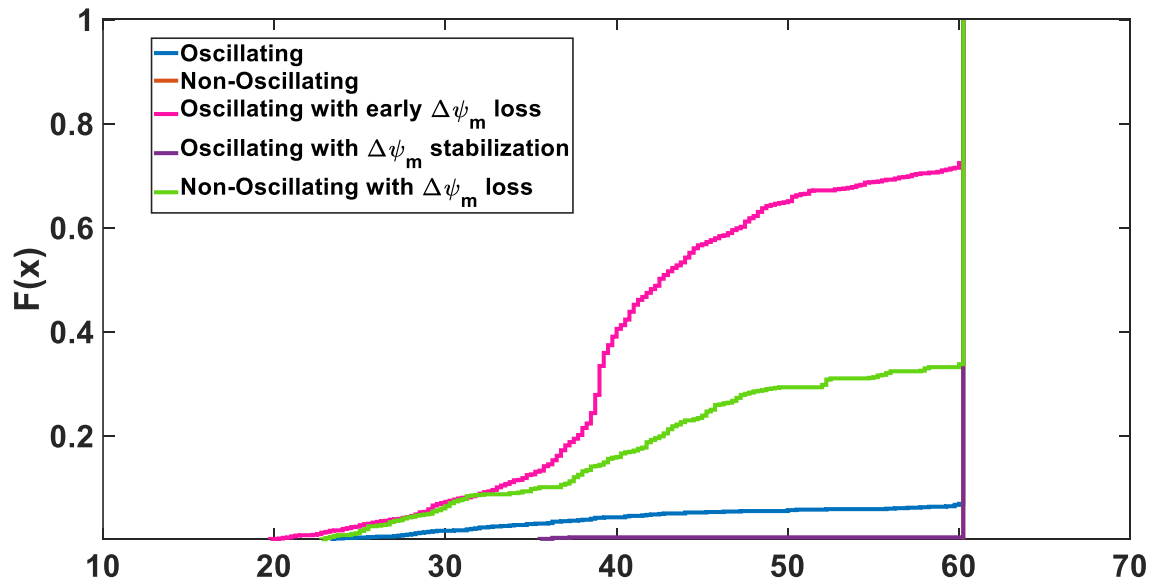

Figure 8: Relationship between Ischemic $\Delta \Psi_{\mathrm{m}}$ depolarization time and Oscillatory behavior during Reperfusion A) Empirical Cumulative Distribution functions showing the probability of depolarization $(F(x))$ as a function of time (x) during Ischemia. B) Empirical Cumulative Distribution functions showing the probability of depolarization ( $F(x))$ as a function of time $(x)$ during Reperfusion. Mitochondrial stabilization during reperfusion correlated with late $\Delta \Psi_{\mathrm{m}}$ loss during Ischemia (purple line). 